\title{
Origin and transition of sex determination mechanisms in a gynogenetic hexaploid fish
}

\author{
Xi-Yin $\mathrm{Li}^{1} \cdot$ Xiao-Li Liu ${ }^{1} \cdot$ Yao-Jun Zhu ${ }^{1} \cdot$ Jun Zhang ${ }^{1} \cdot$ Miao Ding ${ }^{1} \cdot$ Ming-Tao Wang ${ }^{1} \cdot$ Zhong-Wei Wang $^{1} \cdot$ Zhi Li $^{1}$ • \\ Xiao-Juan Zhang ${ }^{1} \cdot$ Li Zhou $^{1} \cdot$ Jian-Fang Gui ${ }^{1}$
}

Received: 7 October 2017 / Accepted: 23 December 2017 / Published online: 2 February 2018

(C) The Genetics Society 2018

\begin{abstract}
Most vertebrates reproduce sexually, and plastic sex determination mechanisms including genotypic sex determination (GSD) and environmental sex determination (ESD) have been extensively revealed. However, why sex determination mechanisms evolve diversely and how they correlate with diverse reproduction strategies remain largely unclear. Here, we utilize the superiority of a hexaploid gibel carp (Carassius gibelio) that is able to reproduce by unisexual gynogenesis and contains a rare but diverse proportion of males to investigate these puzzles. A total of 2248 hexaploid specimens were collected from 34 geographic wild populations throughout mainland China, in which 24 populations were revealed to contain 186 males with various incidences ranging from 1.2 to $26.5 \%$. Subsequently, the proportion of temperaturedependent sex determination (TSD) was revealed to be positively correlated to average annual temperature in wild populations, and male incidence in lab gynogenetic progenies was demonstrated to increase with the increasing of larval rearing temperature. Meanwhile, extra microchromosomes were confirmed to play genotypic male determination role as previously reported. Thereby, GSD and TSD were found to coexist in gibel carp, and the proportions of GSD were observed to be much higher than that of TSD in sympatric wild populations. Our findings uncover a potential new mechanism in the evolution of sex determination system in polyploid vertebrates with unisexual gynogenesis ability, and also reveal a possible association of sex determination mechanism transition between TSD and GSD and reproduction mode transition between unisexual gynogenesis and bisexual reproduction.
\end{abstract}

\section{Introduction}

Most vertebrates are gonochoristic, and females and males are determined via two kinds of diverse and plastic mechanisms, such as genotypic sex determination (GSD) and environmental sex determination (ESD) (Bachtrog et al. 2014; Graves 2008; Mei and Gui 2015). Diverse systems of

$\mathrm{Xi}-\mathrm{Yin} \mathrm{Li}$ and Xiao-Li Liu contributed equally to this work.

Electronic supplementary material The online version of this article (https://doi.org/10.1038/s41437-017-0049-7) contains supplementary material, which is available to authorized users.

Jian-Fang Gui

jfgui@ihb.ac.cn

1 State Key Laboratory of Freshwater Ecology and Biotechnology, Institute of Hydrobiology, Chinese Academy of Sciences,

University of the Chinese Academy of Sciences, Wuhan 430072, China
GSD (Bachtrog et al. 2014) including male heterogametic $\mathrm{XX} / \mathrm{XY}$ system, female heterogametic ZZ/ZW system and their numerous variants were revealed in vertebrates such as mammals (Koopman et al. 1991), birds (Smith et al. 2009), reptiles (Holleley et al. 2015), amphibians (Yoshimoto et al. 2008) and fishes (Matsuda et al. 2002). And different factors for ESD were identified to include temperature (Holleley et al. 2015), photoperiod (Brown et al. 2014), social factors (Warner et al. 1996), $\mathrm{pH}$ and dissolved oxygen (DO) (Baroiller et al. 2009). Although a candidate sex determination gene CIRBP for temperature-dependent sex determination (TSD) was revealed in common snapping turtle (Chelydra serpentina) (Schroeder et al. 2016), most master sex determination genes were illustrated in the pathways of GSD, such as sry in mammals (Koopman et al. 1991), dmy in medaka (Oryzias latipes) (Matsuda et al. 2002), amhy in Nile tilapia (Oreochromis niloticus) (Li et al. 2015) and so on. Sometimes, different sex determination mechanisms were found to coexist in closely related species or different populations of the same species (Bachtrog et al. 2014; 
Holleley et al. 2015). For instance, zebrafish (Danio rerio) has ZZ/ZW system with a strong sex-linked single nucleotide polymorphism (SNP) in wild strains (Wilson et al. 2014), while the domesticated strains use a multigenic sex determination system (Anderson et al. 2012) and also have temperature effect on sex determination (Abozaid et al. 2011). Studies on the half-smooth tongue sole (Cynoglossus semilaevis) suggest that dmrtl is the male determination gene for GSD (Chen et al. 2014; Cui et al. 2017) and epigenetic modification is essential for TSD (Shao et al. 2014). Along with extensive genomic and chromosomal anatomy, labile sex determination systems and diverse sex determination mechanisms have been extensively revealed in many taxa (Aanen et al. 2016; Bachtrog et al. 2014), but why so many ways to evolve diverse sex determination mechanisms and what is the key factor for transition of different sex determination mechanisms remain still unknown.

Gibel carp (Carassius gibelio) with two rounds of polyploidy origins is a recurrent polyploidy hexaploid form of Carassius species complex (Li et al. 2014; Luo et al. 2014; Zhou and Gui 2017), and is able to reproduce by unisexual gynogenesis (Gui and Zhou 2010, 2012). It has a wide geographic distribution in the Eurasian continent and neighboring islands (Gao et al. 2012; Liu et al. 2017a, b). In contrast to other unisexual vertebrates (Neaves and Baumann 2011), rare but significant male incidences were observed in many natural habitats (Gui and Zhou 2010; Jiang et al. 2013). Intriguingly, dual reproduction modes, including unisexual gynogenesis and sexual reproduction, were demonstrated early in 2000 (Gui and Zhou 2010; Zhang et al. 2015; Zhou et al. 2000). Recently, extra microchromosomes with repetitive sequences and transposable elements in males were discovered to play male determination role in the bisexually reproduced progenies ( $\mathrm{Li}$ et al. 2016a). These findings suggest that hexaploid gibel carp might be under the evolutionary trajectory of the reproduction mode transition from unisexual to bisexual reproduction (Li et al. 2016a, 2017 Zhang et al. 2015), and therefore make it an ideal model to investigate evolutionary association between diverse sex determination mechanisms and different reproduction strategies, and to seek the key switch for sex determination mechanism transitions in vertebrates.

Here, we found out that males of gibel carp were not only determined by GSD but also by TSD via analyses of natural populations and lab experimental progenies. Meanwhile, high larval rearing temperature and extra microchromosomes were revealed to play environmental and genotypic male determination role, respectively. Moreover, the proportions of GSD were much higher than that of TSD in sympatric wild populations. And a close association between sex determination mechanism transition and reproduction mode transition was revealed in gibel carp.

\section{Materials and methods}

\section{Ethics statement and experimental fish source}

Animal experiments were conducted in accordance with the regulations of the Guide for Care and Use of Laboratory Animals and were approved by the animal care and use committee of the Institute of Hydrobiology, Chinese Academy of Sciences.

Some various gynogenetic strains of gibel carp $(C$. gibelio) collected from wild populations were named A, B, $\mathrm{C}, \mathrm{D}$ and so on, according to biological characters or genetic markers (Yang et al. 2001; Zhou and Gui 2002). And these strains were used to culture main strains with advantages in aquaculture such as strain DA and strain $\mathrm{A}^{+}$, which were generated by artificial reproduction and maintained through successive gynogenesis (Wang et al. 2011; Zhou and Gui 2002; Zhou et al. 2000). Artificially cultured gibel carp and common carp (Cyprinus carpio) were collected from Guanqiao Experimental Station of Institute of Hydrobiology, Chinese Academy of Sciences, which is located in Wuchang district, Wuhan, China.

Wild samples were collected throughout mainland China as described (Liu et al. 2017b), and the sex was examined by dissection. Detailed information of each locality is listed in Supplementary Table S1. Moreover, four live wild gibel carp including two females $(\mathrm{QI}(-)$ and $९ \mathrm{II}(-))$ and two males $\left(\sigma^{\prime} \mathrm{I}(+)\right.$ and $\left.\mathrm{o}^{\prime} \mathrm{II}(-)\right)$ were sampled from Poyang Lake to perform lab experiments. The phenotypic sex of these fish were distinguished based on whether they ovulated and released eggs or produced sperm in the breeding season. Symbols of "+" and "-" were respectively referred to the presence and absence of the male-specific marker (MSM) identified previously ( $\mathrm{Li}$ et al. 2016a).

Live wild gibel carp sampled from Poyang Lake ( $\mathrm{I}(-)$, OII (-), ơ $\mathrm{I}(+)$, and $\left.\mathrm{o}^{\prime} \mathrm{II}(-)\right)$ were used to perform two parallel lab experiments to confirm applicability of the MSM in wild populations. Strain DA, strain $\mathrm{A}^{+}$, and a wild strain of Poyang Lake (strain PY) were used to reproduce by unisexual gynogenesis to observe temperature effect on sex determination. Strain $\mathrm{A}^{+}$was used to confirm the association between extra microchromosomes and genotypic male determination.

\section{Genomic DNA extraction and PCR detection}

Genomic DNA was extracted from a small piece of fin for each sampled fish by Wizard Genomic DNA Purification Kit (Promega) following the manufacture's protocol as described (Dan et al. 2013; Wang et al. 2009a). MSM was obtained by the primer pair $C g$-MSM-F and $C g$-MSM-R in Taq DNA Polymerase (Fermentas) $20 \mu \mathrm{l}$ reaction mix as described previously ( $\mathrm{Li}$ et al. 2016a). PCR cycling 
a
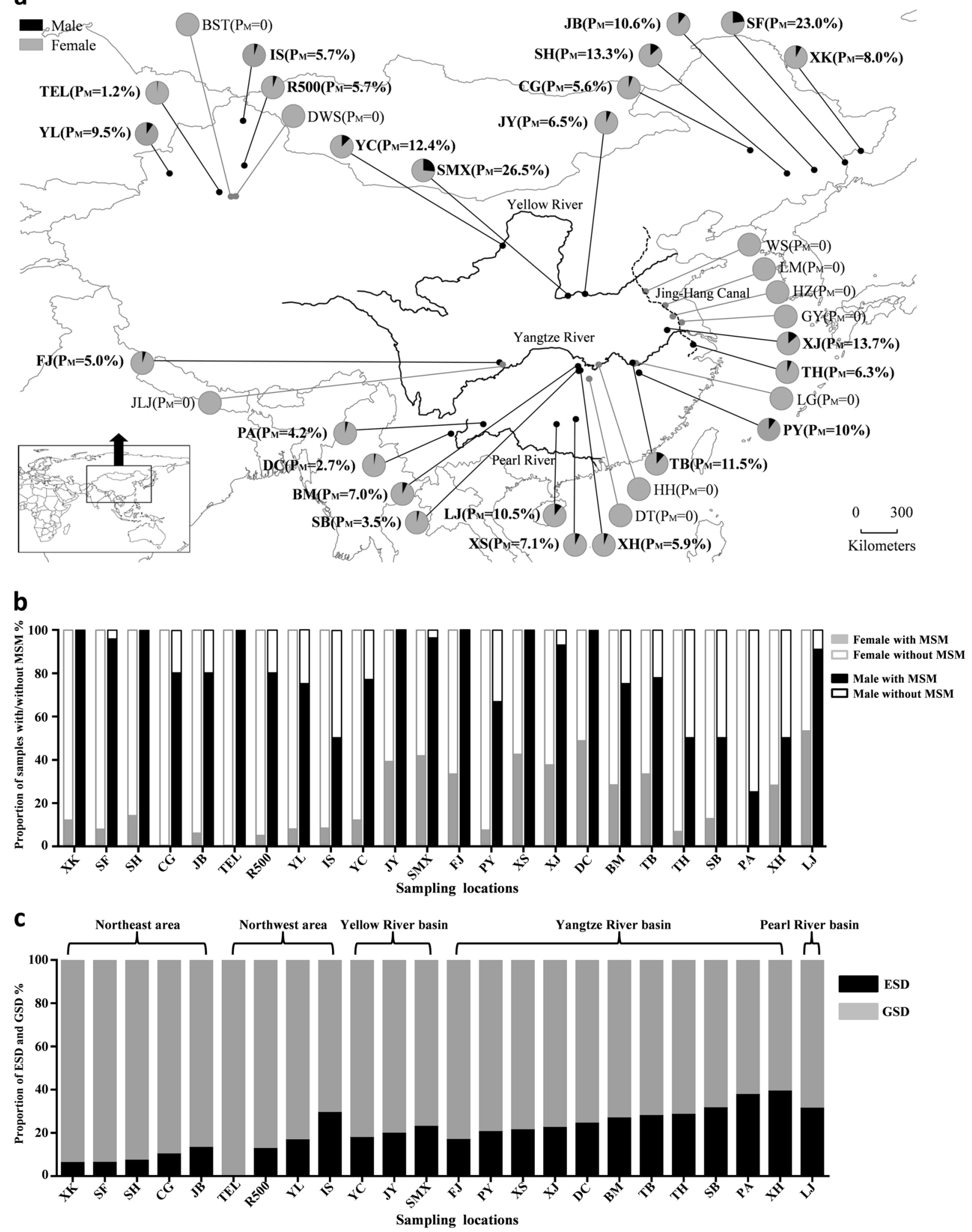

conditions were: $94^{\circ} \mathrm{C}$ for $4 \mathrm{~min} ; 35$ cycles of $94^{\circ} \mathrm{C}$ for 30 $\mathrm{s}, 58^{\circ} \mathrm{C}$ for $30 \mathrm{~s}, 72^{\circ} \mathrm{C}$ for $30 \mathrm{~s} ; 72^{\circ} \mathrm{C}$ for $10 \mathrm{~min}$; endless 4 ${ }^{\circ} \mathrm{C}$. And the amplified sequences from both males and females in wild populations were verified to be the same as the previous MSM sequence (GenBank accession number: KT260068). 
Fig. 1 Coexistence of GSD and ESD in wild populations. a Male incidences in wild populations across mainland China. The proportions of males and females are shown by the areas of black and gray pie charts, respectively. Male proportion of each population is given near the corresponding code. PM: the proportion of males. Detail data are given in Supplementary Table S1, related to (a). b The proportion of samples with/without MSM in females and males in the 24 wild geographic populations with males. c Proportions of GSD and ESD in each population. The geographical groups of these populations are exhibited on the top of the columns. Detail data are given in Supplementary Table S2, related to (b and $\mathbf{c}$ )

\section{Artificial breeding and fish culture}

In the breeding season, the selected maternal fish were induced into spawning by intraperitoneal injection with a mixture of acetone-dried carp pituitary, human chorionic gonadotropin and luteinizing hormone releasing hormone as described previously (Sun et al. 2010). About 8-10 h after injection, the experimental fish started to ovulating, and the ovulated eggs were inseminated with sperms from common carp or gibel carp. The embryos were incubated in culture dishes at $23^{\circ} \mathrm{C}\left( \pm 1^{\circ} \mathrm{C}\right)$ during the periods of embryogenesis and larval hatching, and then the larvae were reared at $20^{\circ} \mathrm{C}$ $\left( \pm 1^{\circ} \mathrm{C}\right)$ in water boxes equipped with an inflator pump. The larvae were fed with fairy shrimp for 35 days since first feeding and then maintained in outdoor tanks $(5 \mathrm{~m} \times 4 \mathrm{~m} \times$ $1.5 \mathrm{~m}$ ) with normal feed. The phenotypic sex of the offspring was distinguished based on whether they ovulated and released eggs or produced sperm during the breeding season in the following year.

For the analysis of temperature influence on male determination, the embryos of the same gynogenetic family were firstly incubated in culture dishes at $23^{\circ} \mathrm{C}\left( \pm 1^{\circ} \mathrm{C}\right)$ until first feeding, and then the larvae were divided into several groups and the raising temperature were gradually changed to $16( \pm 1), 19( \pm 1), 22( \pm 1), 25( \pm 1), 28( \pm 1)$, and 31 $( \pm 1)^{\circ} \mathrm{C}$, respectively. In order to reduce the injury to larvae, the process of temperature changing took about 3-5 h. Subsequently, the larvae were reared in water boxes equipped with inflator pump and under normal photoperiod during the larval rearing period for 35 days. At last the fry were also maintained in outdoor tanks with normal feed.

\section{Statistical analysis}

Pearson correlation analysis was used to estimate the correlation between the proportion of ESD and the environmental stimuli including average annual temperature, $\mathrm{pH}$ and dissolved oxygen via software SPSS v19.0.0. The data of average annual temperature throughout China were collected from WorldClim database (Hijmans et al. 2005) and the data of $\mathrm{pH}$ and dissolved oxygen were obtained from datacenter of ministry of environmental protection of the people's republic of China (http://datacenter.mep.gov.cn/), Lake-Watershed Science Data Center (http://lake.geodata. $\mathrm{cn}$ ), published papers and field measurements. The proportion of temperature sex effect (TSE) against ambient average annual temperature was estimated by applying linear regression using SPSS software v19.0.0.

\section{Chromosome preparation and fluorescence in situ hybridization (FISH)}

Chromosome preparations were performed via the method of head-kidney cell-phytohemagglutinin (PHA) cultivation in vivo as described previously (Zhu et al. 2006). Purified male-specific fragment of gibel carp (Li et al. 2016a) was labeled with Biotin-16-dUTP by Biotin-Nick Translation Mix (Roche) and used as the probe. FISH analysis was performed exactly according to the method described previously (Li et al. 2016a). The microchromosome number was obtained from statistical data of 3 individuals, and 100 metaphases were counted for each tested individual. And the most frequent number was defined as the number of microchromosomes.

\section{Results}

\section{Coexistence of GSD and ESD mechanisms}

A total of 2248 specimens of gibel carp were collected and confirmed as hexaploids by flow cytometry as described (Liu et al. 2017b) from 34 geographic wild populations throughout mainland China, in which 24 populations with male incidences ranging from 1.2 to $26.5 \%$ were revealed to contain 186 males (Fig. 1a and Supplementary Table S1). To unveil the male occurrence reason, we used the recently identified MSM in gibel carp (Li et al. 2016a) to examine these males in the 24 wild populations. Unexpectedly, the MSM was not detected in all of the males, in which the MSM existed in most $(87.1 \%)$ of the males, but it was absent in $12.9 \%$ males. Moreover, we used the MSM to examine all females, and observed that it was absent in most of females $(78.4 \%)$ but existed also in a part of females (21.6\%) (Fig. 1b and Supplementary Table S2). As the MSM was demonstrated to be strictly associated with males in some strains of gibel carp (Li et al. 2016a; Wang et al. 2009b), the inconsistency between the MSM and sex in natural populations implied that two different sex determination mechanisms including GSD and ESD might coexist in the wild populations. And the males without MSM and females with MSM might be the consequence of ESD. Thereby, we defined the ESD proportion as half sum of 


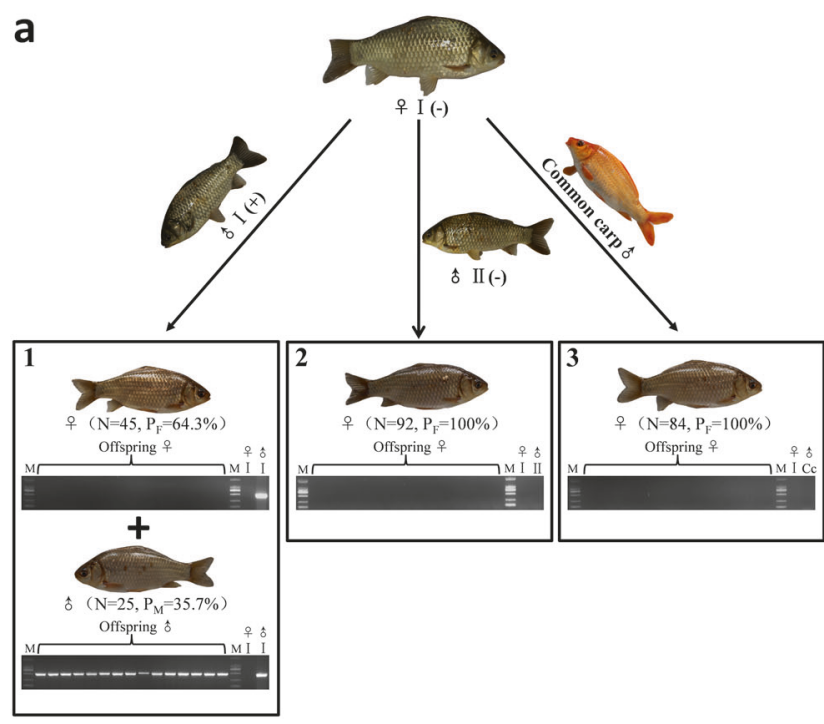

Fig. 2 Confirmation of males resulted from GSD and ESD. a The maternal gibel carp \&I(-) was mated with gibel carp ơ I(+) (1), gibel carp ơII(-) (2), and common carp ơ (3), respectively. b The maternal gibel carp OII $(-)$ was mated with gibel carp ơ $\mathrm{I}(+)(1)$, gibel carp ơlI (-) (2), and common carp ơ (3), respectively. PCR detection of MSM

male proportion without MSM and sympatric female proportion with MSM (ESD proportion $=($ male proportion without MSM + female proportion with MSM)/2). Meanwhile, the half sum of male proportion with MSM and sympatric female proportion without MSM was defined as GSD proportion $(G S D$ proportion $=($ male proportion with $\mathrm{MSM}+$ female proportion without MSM)/2) (Fig. 1c and Supplementary Table S2). Thus, GSD and ESD coexisted in almost all wild populations with males except one population of the TEL, and the proportions of GSD were much higher than that of ESD in sympatric populations (Fig. 1c and Supplementary Table S2).

To confirm that the males with and without MSM were resulted from two different sex determination mechanisms, we selected two females without MSM ( I I(-) and $\mathrm{PII}(-))$, one male with MSM $\left(\mathrm{O}^{\top} \mathrm{I}(+)\right)$ and one male without MSM (o'II(-)) from Poyang Lake population to perform two parallel lab experiments. In the offspring of $\mathrm{I}$ (-) and $\mathrm{OII}(-)$ inseminated by the sperm of $\mathrm{o}^{7} \mathrm{I}(+), 35.7$ and $20.0 \%$ males were generated, respectively, and the MSM was present in all sampled male offspring and the paternal individual, whereas it was absent in all randomly picked female offspring and the maternal individual (Fig. 2a1, b1). However, in the offspring of $\mathrm{II}(-)$ and OII(-) inseminated by the sperm of OrII $\left.^{\prime}-\right)$, no male individuals were observed, and the MSM was also absent in the offspring and the parental individuals (Fig. 2a2, b2), which was similar to the results of gynogenesis stimulated by the sperm of common carp (Fig. 2a3, b3). These results indicate that $\mathrm{O}^{\prime} \mathrm{I}(+)$ is a genotypic male and its

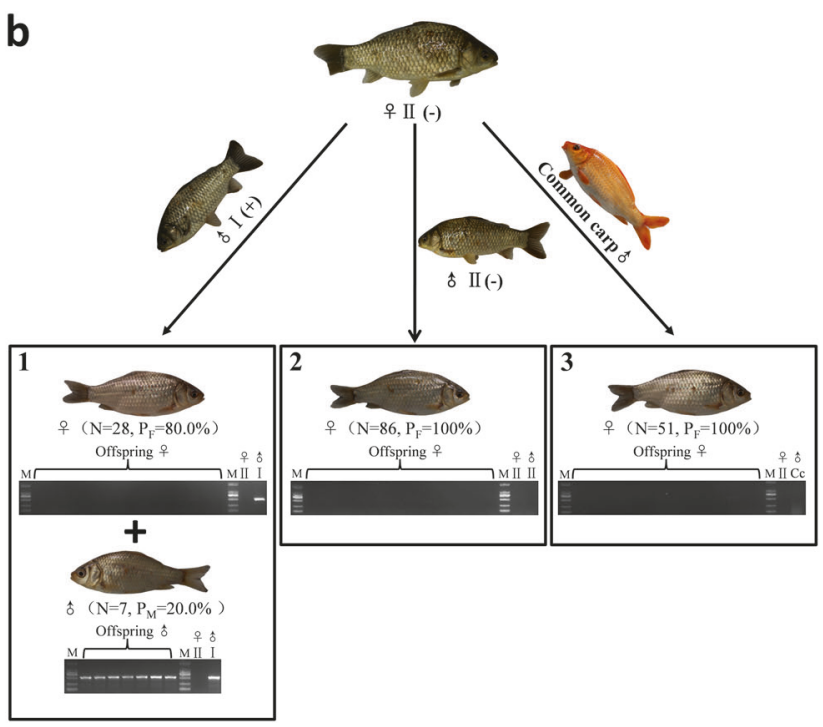

in the offspring and the parental individuals was shown at the bottom. O: female; ơ: male; $(+)$ : with MSM; (-): without MSM; N number of offspring, PM proportion of male offspring, PF proportion of female offspring, Cc Common carp, M: DL2000 marker

sex is determined by GSD, whereas ơII(-) is not a genotypic male and its sex might be determined or affected via ESD.

\section{Average annual temperature is critical factor for ESD in wild populations}

In order to reveal critical factor for ESD, three environmental variables with the ability to determine or influence sex (Baroiller et al. 2009), including average annual temperature (T), $\mathrm{pH}$ and dissolved oxygen (DO), were used to check the correlation with the proportion of ESD (Supplementary Table S3). Correlation analysis showed that the proportion of ESD was only significantly correlated to the average annual temperature (Pearson correlation $=0.770, P$ $<0.001$ ) (Supplementary Table S4), suggesting that TSE should be critical for the ESD. Intriguingly, the proportions of TSE were variable, ranging from 0 (TEL) to $39.1 \%$ (XH) at different localities throughout mainland China (Fig. 3a). And the average proportions of TSE in northeast of China (8.4\%), northwest of China (14.6\%), Yellow River basin (20.0\%), Yangtze River basin (26.9\%) and Pearl River basin $(31.2 \%)$ increased orderly from north to south with the increasing of average annual temperature in these areas (Fig. 3a, b). And, the proportion of TSE in each population against the average annual temperature showed significant positive correlation via linear regression analysis (Fig. 3c). These data suggest that temperature might be the critical factor for ESD of the wild populations, and TSD should exist in gibel carp. 


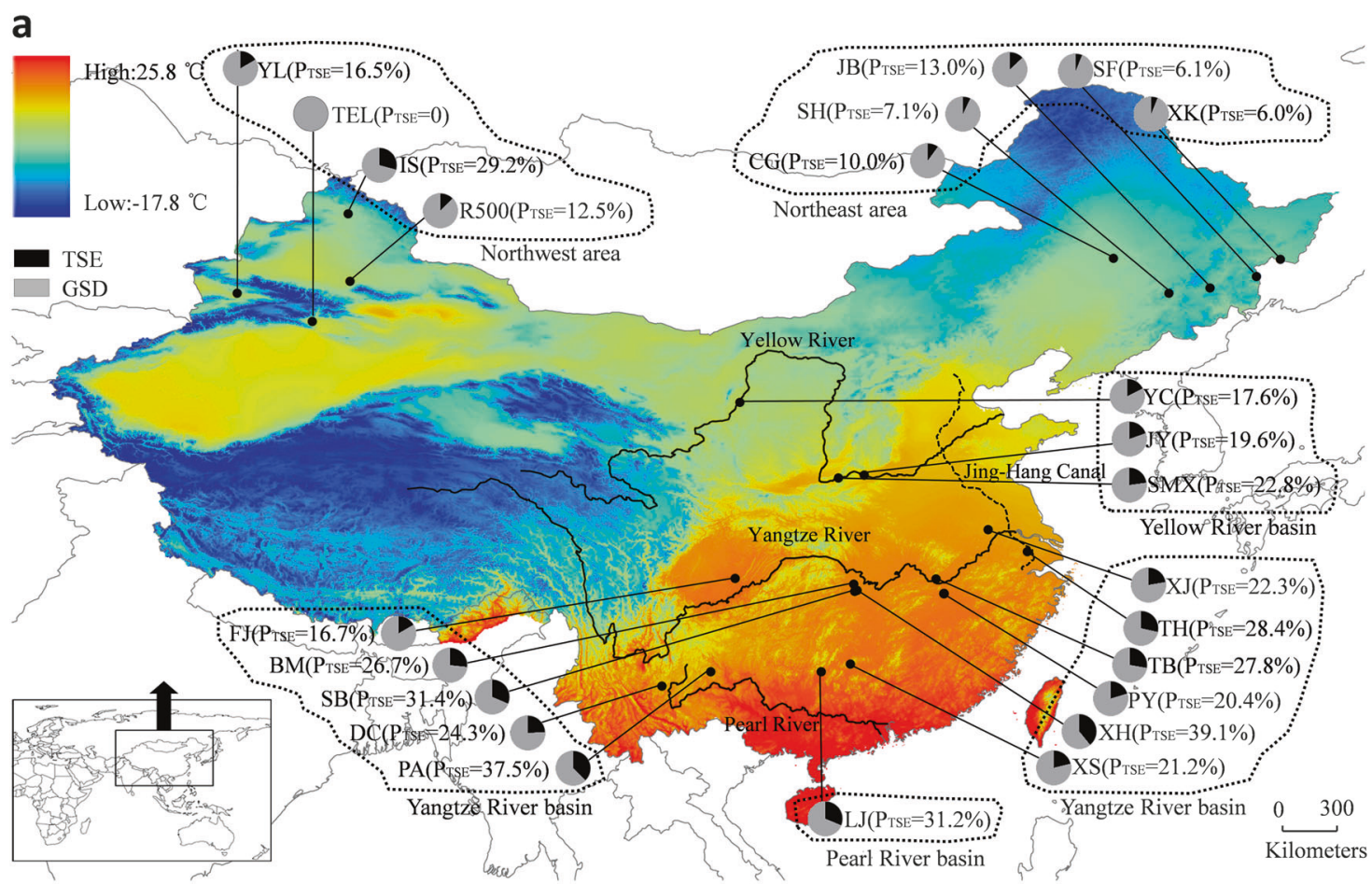

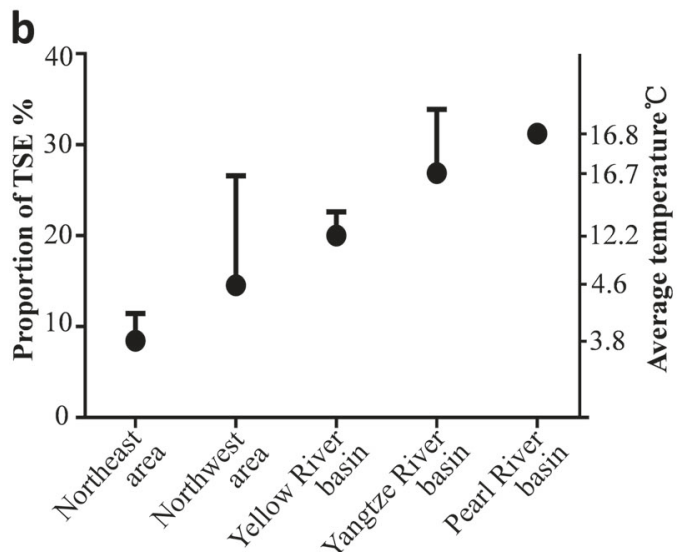

Geographic area

Fig. 3 Correlation between proportion of temperature sex effect (TSE) and ambient temperature. a The proportions of TSE and GSD are shown by the areas of black and gray pie charts, respectively. $P_{\mathrm{TSE}}$ the proportion of TSE in each population. The dashed boxes indicate geographic groups of these populations. b Average proportions of TSE in five geographic groups through mainland China. Geographic area is exhibited on the $X$-axis. The average proportion of TSE is indicated on

\section{High rearing temperature plays environmental male determination role}

To confirm existence of TSD in gibel carp, we utilized the superiority of unisexual gynogenesis with genotypic allfemale larvae (Gui and Zhou 2010; Li et al. 2016a; Zhang et al. 2015) to establish three different gynogenetic families from two artificial strains (strain $\mathrm{A}^{+}$and strain DA) and a wild strain of Poyang Lake (strain PY), and reared them in

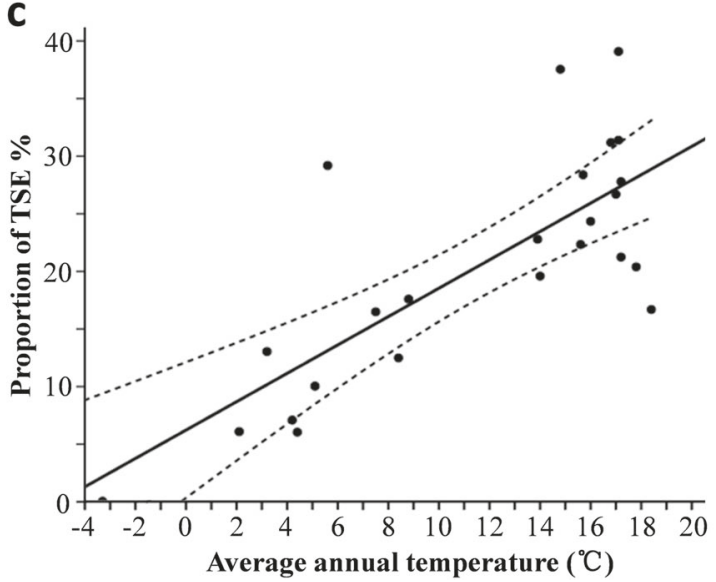

the left $Y$-axis, and average temperature of corresponding geographic area is showed on the right $Y$-axis. Vertical bars: standard deviation of each area. c Linear regression analysis of the proportion of TSE in each locality against the ambient average annual temperature. The solid line is the fitted line derived from the black dots which represent the data of 24 wild populations with males. The region between dashed lines indicates $95 \%$ confidence limits

different temperatures of $16,19,22,25,28$, and $31^{\circ} \mathrm{C}$ to observe temperature effect on sex determination (Fig. 4a). Although male proportions are different among the three gynogenetic families of $\mathrm{A}^{+}$, DA, and PY, their male occurrence proportions always increased along with increasing of the rearing temperature. And correlation analyses showed that male proportions were all significantly correlated to larval rearing temperature in strain $\mathrm{A}^{+}$(Pearson $\quad$ correlation $=0.906, \quad P<0.001)$, DA $\quad$ (Pearson 
Fig. 4 Male proportion in gynogenetic offspring as a function of larval rearing temperature. a Schematic diagram for the analysis of temperature influence on male determination. O: female; on: male. b Male proportions in gynogenetic offspring of two artificial strains (strain $\mathrm{A}^{+}$and strain DA) and a wild strain (strain PY) are indicated by circles, squares and triangles, respectively. Larval rearing temperature is shown on the $X$ axis, and male proportion in gynogenetic offspring is exhibited on the $Y$-axis. Vertical bars: standard deviation of each temperature. Detail data are given in Supplementary Table S5, related to (b)

\section{a}

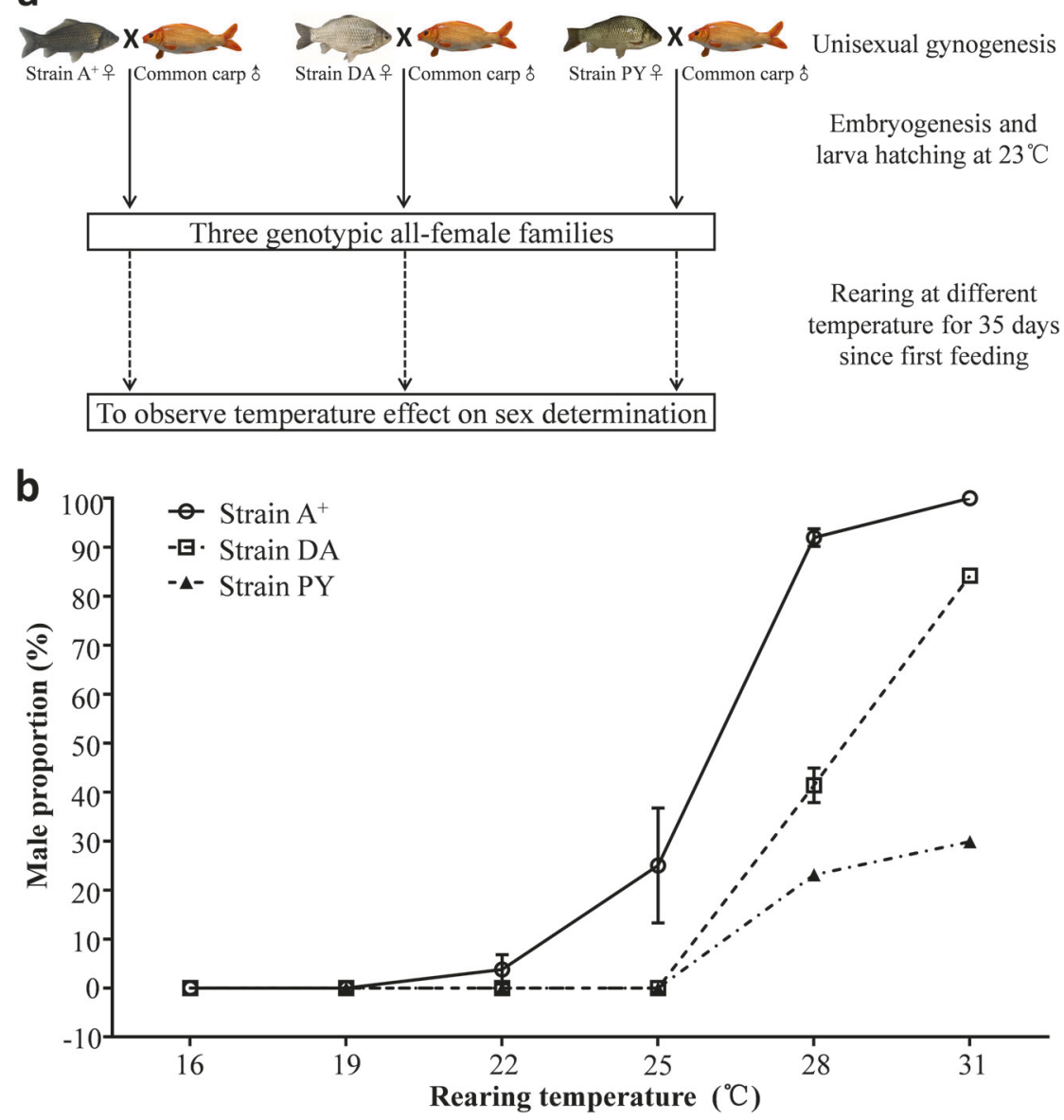

correlation $=0.841, P<0.005)$, and PY (Pearson correlation $=0.891, P<0.05)$. In strain $\mathrm{A}^{+}$, all-females were produced when the temperature was lower than $19^{\circ} \mathrm{C}$, while males started to emerge in the gynogenetic family when the temperature was over $22^{\circ} \mathrm{C}$, and all-male individuals were generated when the temperature was higher than $31^{\circ} \mathrm{C}$. In strain DA and strain PY, all-female individuals were produced when the temperature was lower than $25^{\circ} \mathrm{C}$, while males started to be generated when the temperature was higher than $28^{\circ} \mathrm{C}$ (Fig. $4 \mathrm{~b}$ and Supplementary Table S5). The lab experimental data certify that high rearing temperature plays male determination role in the three gynogenetic families.

\section{Extra microchromosomes play genotypic male determination role}

Extra microchromosomes with repetitive sequences and transposable elements in males were revealed to play genotypic male determination role in strain DA of gibel carp previously ( $\mathrm{Li}$ et al. 2016a). Here we further analyzed extra microchromosomes of genotypic males in a bisexually reproduced family from strain $\mathrm{A}^{+}$. One hundred metaphases were counted for each tested individual, and statistical data from three males of GSD revealed that $30.7 \%( \pm 3.1 \%)$ metaphases contained the most frequent microchromosome number of 17 (Fig. 5a, b, left), which indicated that males of GSD have 17 microchromosomes. Meanwhile, both $40.7 \%$ $( \pm 11.7 \%)$ metaphases from males of TSD (Fig. 5a, b, middle) and $47.3 \%( \pm 14.6 \%)$ metaphases from females (Fig. 5a, b, middle) had the most frequent microchromosome number of 9, which suggested that there were nine microchromosomes in males of TSD and in females, respectively. In comparison with males of TSD and females, the metaphases of genotypic males have eight extra microchromosomes (Fig. 5), suggesting that the extra microchromosomes might be associated with genotypic male determination in strain $\mathrm{A}^{+}$as in stain DA ( $\mathrm{Li}$ et al. 2016a).

\section{Discussion}

The current investigation provides new relevant evidences on the coexistence of genotypic and TSD and the origin and transition of sex determination mechanisms in a hexaploid 
a
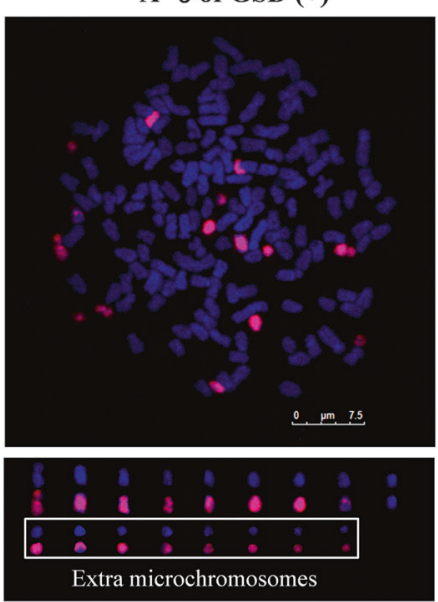

b

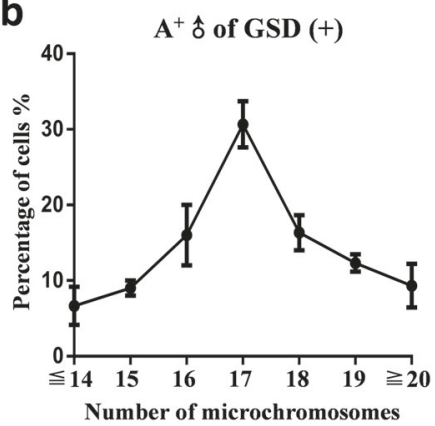

$\mathrm{A}^{+}$ô of TSD (-)
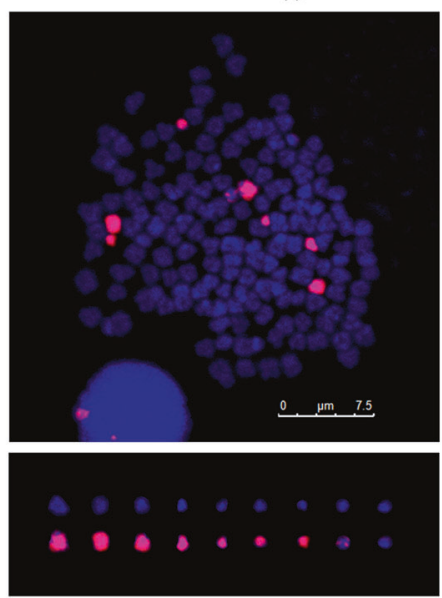

$\mathrm{A}^{+}$ô of TSD (-)

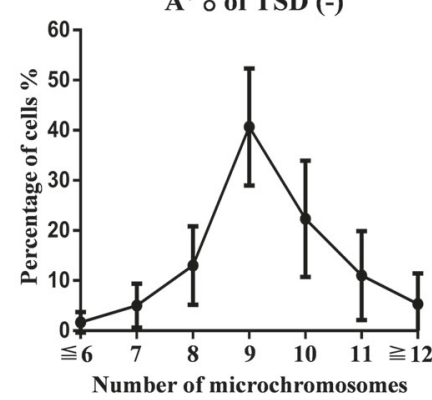

$\mathbf{A}^{+}$우(-)
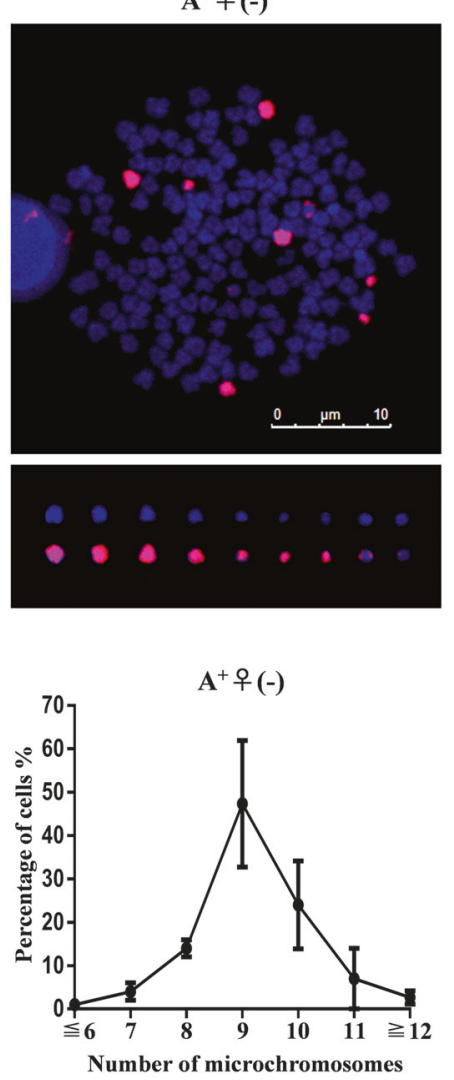

Fig. 5 Extra microchromosomes in males of GSD. a FISH analysis in metaphase of a male of GSD (left), a male of TSD (middle) and a female (right) from strain $\mathrm{A}^{+}$. Microchromosomes in the corresponding metaphase are exhibited at the bottom, and extra microchromosomes in the male of GSD are indicated in the white box. The probe was labeled with Biotin and red fluorescence was produced. All metaphase chromosomes were counterstained with DAPI and appeared as blue. b Statistical data of microchromosome number in males of GSD (left), males of TSD (middle) and females (right) from strain $\mathrm{A}^{+}$. Three individuals were examined for each kind of fish, and a total of 100 metaphases were counted for each tested individual. Number of microchromosomes is shown on the $X$-axis, and the percentage of total cells is shown on the $Y$-axis. O: female; ơ: male; $(+)$ : with MSM; $(-)$ : without MSM gibel carp by detecting the presence of male-specific marker and extra microchromosomes in males reported previously (Li et al. 2016a, 2017). And, the evidences come from wild population analyses collected across mainland China, and also from experimental family progeny demonstration raising individual broods at different temperatures. Our observation that TSD and GSD coexist in the hexaploid gibel carp implies that different sex determination mechanisms have originated in the extant polyploid fish that has undergone recurrent polyploidy ( $\mathrm{Li}$ et al. 2014; Liu et al. 2017a, b) and possesses unisexual gynogenesis ability (Gui and Zhou 2010; Zhang et al. 2015). Both TSD and GSD are widespread in natural habitats of gibel carp across mainland China (Figs. 1 and 3), however, the proportion of GSD is much higher than that of TSD in sympatric wild populations especially in the localities with low average annual temperature (Figs. 1 and 3). The dominant presence of GSD in gibel carp might be caused by the selection of sex determination per se, or by the improvement of microchromosome transmission. No matter or what, the extra microchromosomes with genotypic male determination role (Fig. 5) (Li et al. 2016a) might have strong selective benefits and be preserved in the extant hexaploid gibel carp.

On the other hand, hexaploid gibel carp has been demonstrated to acquire facultative reproduction strategies of unisexual gynogenesis and bisexual reproduction (Gui and Zhou 2010; Li et al. 2016a; Zhang et al. 2015; Zhou et al. 2000), in comparison with other polyploid vertebrates with obligate unisexual reproduction modes (Choleva and Janko 2013; Lampert and Schartl 2010; Stock et al. 2012). Unisexual gynogenesis is able to avoid mating costs and achieve high fecundity, and occasional bisexual reproduction is able to prevent the accumulation of deleterious allele combinations and to promote the fixation of beneficial mutations (Burke and Bonduriansky 2017). Thus, the recurrent hexaploid gibel carp can use all the benefits of unisexual gynogenesis and bisexual reproduction and 
outperform both obligate bisexual and obligate unisexual strategies. Perhaps, the facultative reproduction strategies of unisexual and bisexual reproduction modes in hexaploid gibel carp might be related to the wide geographic distribution and high genetic diversity observed previously (Liu et al. 2017a, b). Although some unisexual geckos in the genera Lepidodactylus and Nactus have been revealed to have widespread ecological distribution (Avise 2015) and the oldest unisexual vertebrate salamander Ambystoma has been estimated to be up to 5 million years ( $\mathrm{Bi}$ and Bogart 2010), unisexual reproductions are suggested to be almost invariable failure as a long-term evolutionary strategy (Avise 2015), which may be the reason that most species reproduce by obligate bisexual reproduction (Burke and Bonduriansky 2017; Neaves and Baumann 2011). And for a long-term, the hexaploid gibel carp might be on the evolutionary trajectory from unisexual gynogenesis to bisexual reproduction (Li et al. 2016a; Liu et al. 2017b; Zhang et al. 2015).

The most intriguing finding is the association of sex determination mechanism transition between TSD and GSD and the reproduction mode transition between unisexual gynogenesis and sexual reproduction in the recurrent polyploidy fish. Hexaploid gibel carp has been revealed to undergo two rounds of polyploidy origins ( $\mathrm{Li}$ et al. 2014; Luo et al. 2014), and the early polyploidy event might have resulted in ancestral tetraploid Carassius auratus that has earned bisexual reproductive ability through the diploidization evolution (Li et al. 2014; Liu et al. 2017b; Luo et al. 2007), whereas the late recurrent polyploidy might lead to occurrence of the extant hexaploid gibel carp at around 0.5 million years ago and make it reenter an evolutionary trajectory of diploidization ( $\mathrm{Li}$ et al. 2014; Liu et al. 2017a, b). The newly formed hexaploid gibel carp might reproduce via unisexual gynogenesis, as polyploidy consequences are frequently related to clonal reproduction modes, such as gynogenesis, parthenogenesis, hybridogenesis and kleptogenesis (Choleva and Janko 2013; Lampert and Schartl 2010; Li et al. 2016b; Stock et al. 2012). Gynogenetic populations have nearly identical genetic background (Gui and Zhou 2010; Zhang et al. 2015), which do not fill the criteria for the assignment of GSD (Ospina-Alvarez and Piferrer 2008; Valenzuela et al. 2003), so exclusive TSD might exist in the hexaploid gibel carp with unisexual gynogenesis. During the diploidization process, GSD with evolutionary adaptions have been selected in the extant hexaploid gibel carp, and transition between TSD and GSD might associate with transition between unisexual gynogenesis and bisexual reproduction, as males of GSD are able to produce genotypic male offspring similar to bisexual reproduction while males of TSD can only produce allfemale offspring like typical unisexual gynogenesis (Fig. 2).
Although female with MSM was detected in lab experiments occasionally and could produce a high proportion of male offspring (Wang et al. 2009b), it is still unclear why there are quite a few females with MSM in wild populations. One explanation is that GSD associated with MSM may be not always dominant to TSD, especially in some populations with high sensitivity of ambient temperature. And we also cannot exclude another explanation that there might be other unidentified genotypic and/or ESD mechanisms which are not associated with MSM, as hexaploid gibel carp has multiple independent polyploidy origins from sympatric tetraploid $C$. auratus across China (Liu et al. 2017a, b; Luo et al. 2014).

In conclusion, significant and variable male incidences were observed in natural habitats of gibel carp. Subsequently, through analyses of natural populations and lab experimental progenies, we found out that males of gibel carp were determined via not only genetic but also environmental factors, and these factors were illustrated to be extra microchromosomes and larval rearing temperature, respectively. Moreover, our data indicated that GSD might possess stronger ecological and evolutionary adaption than TSD, and there might be a close association between sex determination mechanism transition and reproduction mode transition in gibel carp. Therefore, this study gives a hint for understanding evolutionary adaptation and transition of sex determination mechanisms and reproduction strategies.

\section{Data accessibility}

All relevant data are within the paper and its Supplementary materials.

\section{Data archiving}

This article does not report new empirical data or software.

Acknowledgements This work was supported by the Key Program of Frontier Sciences of the Chinese Academy of Sciences (QYZDYSSW-SMC025), the National Natural Science Foundation of China (31502148), the Strategic Priority Research Program of the Chinese Academy of Sciences (XDA08030201), the Young Elite Scientist Sponsorship Program by CAST (YESS20150156), the Earmarked Fund for Modern Agro-industry Technology Research System (NYCYTX-49), the Autonomous Project of the State Key Laboratory of Freshwater Ecology and Biotechnology (2016FBZ01), the Autonomous Project of the Institute of Hydrobiology, Chinese Academy of Sciences (Y25A171) and the Knowledge Innovation Program of the Chinese Academy of Science. We would like to thank Fang Zhou for providing confocal services (Analytical \& Testing Center, IHB, CAS).

\section{Compliance with ethical standards}

Conflict of interest The authors declare that they have no conflict of interest. 


\section{References}

Aanen D, Beekman M, Kokko H (2016) Weird sex: the underappreciated diversity of sexual reproduction. Philos Trans R Soc B 371:4

Abozaid H, Wessels S, Hörstgenschwark G (2011) Effect of rearing temperatures during embryonic development on the phenotypic sex in zebrafish (Danio rerio). Sex Dev 5:259-265

Anderson JL, Marí AR, Braasch I, Amores A, Hohenlohe P, Batzel P et al. (2012) Multiple sex-associated regions and a putative sex chromosome in zebrafish revealed by RAD mapping and population genomics. PLoS One 7:e40701

Avise JC (2015) Evolutionary perspectives on clonal reproduction in vertebrate animals. Proc Natl Acad Sci USA 112:8867-8873

Bachtrog D, Mank JE, Peichel CL, Kirkpatrick M, Otto SP, Ashman TL et al. (2014) Sex determination: why so many ways of doing it? PLoS Biol 12:e1001899

Baroiller JF, D'Cotta H, Saillant E (2009) Environmental effects on fish sex determination and differentiation. Sex Dev 3:118-135

Bi K, Bogart JP (2010) Time and time again: unisexual salamanders (genus Ambystoma) are the oldest unisexual vertebrates. BMC Evol Biol 10:14

Brown EE, Baumann H, Conover DO (2014) Temperature and photoperiod effects on sex determination in a fish. J Exp Mar Biol Ecol 461:39-43

Burke NW, Bonduriansky R (2017). Sexual conflict, facultative asexuality, and the true paradox of sex. Trends Ecol Evol. https:// doi.org/10.1016/j.tree.2017.06.002.

Chen S, Zhang G, Shao C, Huang Q, Liu G, Zhang P et al. (2014) Whole-genome sequence of a flatfish provides insights into $\mathrm{ZW}$ sex chromosome evolution and adaptation to a benthic lifestyle. Nat Genet 46:253-260

Choleva L, Janko K (2013) Rise and persistence of animal polyploidy: evolutionary constraints and potential. Cytogenet Genome Res 140:151-170

Cui Z, Liu Y, Wang W, Wang Q, Zhang N, Lin F et al. (2017) Genome editing revealsdmrtl as an essential male sexdetermining gene in Chinese tongue sole (Cynoglossus semilaevis). Sci Rep 7:42213

Dan C, Mei J, Wang D, Gui JF (2013) Genetic differentiation and efficient sex-specific marker development of a pair of Yand $\mathrm{X}$-linked markers in yellow catfish. Int $\mathrm{J}$ Biol Sci 9:1043-1049

Gao Y, Wang SY, Luo J, Murphy RW, Du R, Wu SF et al. (2012) Quaternary palaeoenvironmental oscillations drove the evolution of the Eurasian Carassius auratus complex (Cypriniformes, Cyprinidae). J Biogeogr 39:2264-2278

Graves JAM (2008) Weird animal genomes and the evolution of vertebrate sex and sex chromosomes. Annu Rev Genet 42:565-586

Gui JF, Zhou L (2010) Genetic basis and breeding application of clonal diversity and dual reproduction modes in polyploid Carassius auratus gibelio. Sci China Life Sci 53:409-415

Gui JF, Zhu ZY (2012) Molecular basis and genetic improvement of economically important traits in aquaculture animals. Chin Sci Bull 57(15):1751-1760

Hijmans RJ, Cameron SE, Parra JL, Jones PG, Jarvis A (2005) Very high resolution interpolated climate surfaces for global land areas. Int J Climatol 25:1965-1978

Holleley CE, O'Meally D, Sarre SD, Marshall Graves JA, Ezaz T, Matsubara K et al. (2015) Sex reversal triggers the rapid transition from genetic to temperature-dependent sex. Nature 523:79-82

Jiang FF, Wang ZW, Zhou L, Jiang L, Zhang XJ, Apalikova OV et al. (2013) High male incidence and evolutionary implications of triploid form in northeast Asia Carassius auratus complex. Mol Phylogenet Evol 66:350-359

Koopman P, Gubbay J, Vivian N, Goodfellow P, Lovellbadge R (1991) Male development of chromosomally female mice transgenic for Sry. Nature 351:117-121

Lampert KP, Schartl M (2010) A little bit is better than nothing: the incomplete parthenogenesis of salamanders, frogs and fish. BMC Biol 8:78

Li MH, Sun YL, Zhao JE, Shi HJ, Zeng S, Ye K et al. (2015) A tandem duplicate of anti-Mullerian hormone with a missense SNP on the $\mathrm{Y}$ chromosome is essential for male sex determination in Nile tilapia, Oreochromis niloticus. PLoS Genet 11:e1005678

Li XY, Liu XL, Ding M, Li Z, Zhou L, Zhang XJ et al. (2017) A novel male-specific SET domain-containing gene setdm identified from extra microchromosomes of gibel carp males. Sci Bull $62: 528-536$

Li XY, Zhang QY, Li Z, Li Z, Zhang XJ, Wang D et al. (2016a) Extra microchromosomes play male determination role in polyploid gibel carp. Genetics 203:1415-1424

Li XY, Zhang XJ, Li Z, Hong W, Liu W, Zhang J et al. (2014) Evolutionary history of two divergent Dmrt1 genes reveals two rounds of polyploidy origins in gibel carp. Mol Phylogenet Evol 78:96-104

Li Z, Liang HW, Wang ZW, Zou GW, Gui JF (2016b) A novel allotetraploid gibel carp strain with maternal body type and growth superiority. Aquaculture 458:55-63

Liu XL, Li XY, Jiang FF, Wang ZW, Li Z, Zhang XJ et al. (2017a). Numerous mtDNA haplotypes reveal multiple independent polyploidy origins of hexaploids in Carassius species complex. Ecol Evol https://doi.org/10.1002/ece3.3462.

Liu XL, Jiang FF, Wang ZW, Li XY, Li Z, Zhang XJ et al. (2017b) Wider geographic distribution and higher diversity of hexaploids than tetraploids in Carassius species complex reveal recurrent polyploidy effects on adaptive evolution. Sci Rep 7:5395

Luo J, Gao Y, Ma W, Bi XY, Wang SY, Wang J et al. (2014) Tempo and mode of recurrent polyploidization in the Carassius auratus species complex (Cypriniformes, Cyprinidae). Heredity 112:415-427

Luo J, Stadler PF, He SP, Meyer A (2007) PCR survey of Hox genes in the goldfish Carassius auratus auratus. J Exp Zool Part B 308B:250-258

Matsuda M, Nagahama Y, Shinomiya A, Sato T, Matsuda C, Kobayashi T et al. (2002) $D M Y$ is a Y-specific DM-domain gene required for male development in the medaka fish. Nature 417:559-563

Mei J, Gui JF (2015) Genetic basis and biotechnological manipulation of sexual dimorphism and sex determination in fish. Sci China Life Sci 58:124-136

Neaves WB, Baumann P (2011) Unisexual reproduction among vertebrates. Trends Genet 27(3):81-88

Ospina-Alvarez N, Piferrer F (2008) Temperature-dependent sex determination in fish revisited: prevalence, a single sex ratio response pattern, and possible effects of climate change. PLoS One 3:e2837

Schroeder AL, Metzger KJ, Miller A, Rhen T (2016) A novel candidate gene for temperature-dependent sex determination in the common snapping turtle. Genetics 203:557-571

Shao C, Li Q, Chen S, Zhang P, Lian J, Hu Q et al. (2014) Epigenetic modification and inheritance in sexual reversal of fish. Genome Res 24:604-615

Stock M, Ustinova J, Betto-Colliard C, Schartl M, Moritz C, Perrin N (2012) Simultaneous Mendelian and clonal genome transmission in a sexually reproducing, all-triploid vertebrate. Proc R Soc Lond B 279:1293-1299

Sun M, Li Z, Gui JF (2010) Dynamic distribution of spindlin in nucleoli, nucleoplasm and spindle from primary oocytes to 
mature eggs and its critical function for oocyte-to-embryo transition in gibel carp. J Exp Zool Part A 313A:461-473

Valenzuela N, Adams DC, Janzen FJ (2003) Pattern does not equal process: exactly when is sex environmentally determined? Am Nat 161:676-683

Wang D, Mao HL, Chen HX, Liu HQ, Gui JF (2009a) Isolation of Yand X-linked SCAR markers in yellow catfish and application in the production of all-male populations. Anim Genet 40:978-981

Wang D, Mao HL, Peng JX, Li XY, Zhou L, Gui JF (2009b) Discovery of a male-biased mutant family and identification of a male-specific SCAR marker in gynogenetic gibel carp Carassius auratus gibelio. Prog Nat Sci 19:1537-1544

Wang ZW, Zhu HP, Wang D, Jiang FF, Guo W, Zhou L et al. (2011) A novel nucleo-cytoplasmic hybrid clone formed via androgenesis in polyploid gibel carp. BMC Res Notes 4:82

Warner RR, Fitch DL, Standish JD (1996) Social control of sex change in the shelf limpet, Crepidula norrisiarum: size-specific responses to local group composition. J Exp Mar Biol Ecol 204:155-167

Wilson CA, High SK, McCluskey BM, Amores A, Yan YL et al. (2014) Wild sex in zebrafish: loss of the natural sex determinant in domesticated strains. Genetics 198:1291-1308

Yang L, Yang ST, Wei XH, Gui JF (2001) Genetic diversity among different clones of the gynogenetic silver crucian carp, Carassius auratus gibelio, revealed by transferrin and isozyme markers. Biochem Genet 39:213-225
Yoshimoto S, Okada E, Umemoto H, Tamura K, Uno Y, NishidaUmehara $\mathrm{C}$ et al. (2008) A W-linked DM-domain gene, $D M$ - $W$, participates in primary ovary development in Xenopus laevis. Proc Natl Acad Sci USA 105:2469-2474

Zhang J, Sun M, Zhou L, Li Z, Liu Z, Li XY et al. (2015) Meiosis completion and various sperm responses lead to unisexual and sexual reproduction modes in one clone of polyploid Carassius gibelio. Sci Rep 5:10898

Zhou L, Gui J (2017) Natural and artificial polyploids in aquaculture. Aquac Fish 2:103-111

Zhou L, Gui JF (2002) Karyotypic diversity in polyploid gibel carp, Carassius auratus gibelio Bloch. Genetica 115:223-232

Zhou L, Wang Y, Gui JF (2000) Genetic evidence for gonochoristic reproduction in gynogenetic silver crucian carp (Carassius auratus gibelio bloch) as revealed by RAPD assays. J Mol Evol 51:498-506

Zhu HP, Ma DM, Gui JF (2006) Triploid origin of the gibel carp as revealed by $5 \mathrm{~S}$ rDNA localization and chromosome painting. Chromosome Res 14:767-776

Smith CA, Roeszler KN, Ohnesorg T, Cummins DM, Farlie PG, Doran TJ et al (2009) The avian Z-linked gene DMRT1 is required for male sex determination in the chicken Nature 461:267-271 